\title{
Frequency and clinical characteristics of HER2 over-expressed breast cancer in Saudi Arabia: a retrospective study
}

Jamal Zekri ${ }^{*}$, Ahmed Saadeddin ${ }^{2}$ and Hulayel Alharbi ${ }^{3}$

\begin{abstract}
Introduction: This study aimed to determine the frequency of human epidermal growth factor receptor 2 (HER2) over-expression in newly diagnosed breast cancer (BC) patients in Saudi Arabia and to assess the clinical characteristics and outcomes in patients with HER2-positive disease.

Methods: In the first part of the study, we retrospectively reviewed the pathology records of all patients diagnosed with BC between 2007 and 2013 at 3 hospitals in the largest 3 cities in Saudi Arabia to determine the frequency of HER2 over-expression. In the second part, a representative sample from the patients identified with HER2 overexpressed BC was selected for further investigation. Data collected included demographic and clinical characteristics such as hormone-receptor status, treatment regimens, survival data, response to treatment, and selected adverse events.

Results: $1867 \mathrm{BC}$ records were included in the study. HER2 was overexpressed in 559 patients (29.9\%); of those, 348 HER2-positive BC patients were included in subsequent analyses. In the sample of HER2-positive BC patients, median age at diagnosis was 46 years, $0.9 \%$ were male, $92.5 \%$ were Saudi, $42.4 \%$ were Hormone Receptor-negative, and $13.1 \%$ had stage IV tumors. Most patients (84.2\%) underwent curative intent surgery and $71.8 \%$ received radiotherapy. Average tumor size was $3.5 \pm 2.5 \mathrm{~cm}$ and infiltrating ductal carcinoma was the most common pathology (92.9\%). As for pharmacological therapy, the most commonly used regimens were Chemotherapy + Trastuzumab combination (79.1\%) in neoadjuvant setting, Hormonotherapy alone (56.2\%) in adjuvant setting, and Chemotherapy + Targeted therapy combination (64.8\%) as palliative treatment. At the last patient evaluation, 36.9\% had complete response, while 33.2\% had progressive disease. Median overall survival (OS) and progression-free survival (PFS) were not reached in patients on neoadjuvant/adjuvant pharmacotherapy. As for patients on palliative intent pharmacotherapy, median OS and PFS were 64.7 and 29.3 months respectively.
\end{abstract}

Conclusion: This study provided updated figures regarding HER2 overexpression in BC in Saudi Arabia: HER2 overexpression rate (29.9\%) was within the range reported in previous studies. Patients' demographic and clinical characteristics were also similar to those reported earlier, with a median age at diagnosis of 46 years and one third of patients having locally advanced/metastatic disease at diagnosis.

Keywords: Breast cancer, HER2, Saudi Arabia, Epidemiology

*Correspondence: jmzekri@hotmail.com

${ }^{1}$ King Faisal Specialist Hospital and Research Centre, College of Medicine,

Alfaisal University, Jeddah, Saudi Arabia

Full list of author information is available at the end of the article

\section{Background}

Breast cancer $(\mathrm{BC})$ is the second most commonly diagnosed cancer in the world and the most commonly diagnosed cancer and the leading cause of cancer death among females. More than two million new cases of BC 
are diagnosed annually, accounting for almost 1 in 4 cancer cases among women and $15 \%$ of cancer mortality in both developed and developing countries [1].

Age at diagnosis varies substantially between industrialized and Arab countries; in USA and Europe, the median age at presentation is around 63 years [2]. However, in the Arab countries, almost $50 \%$ of the cases are diagnosed at age below 50 (median age at diagnosis is 49.8 years). As for the stage of the disease, $\mathrm{BC}$ is more commonly diagnosed in advanced and metastatic stages in developing than in developed countries [3].

The situation in Saudi Arabia is no exception from other parts of the Arab world, where BC accounts for $30.1 \%$ of female cancers [4]. The Saudi National Cancer Registry reported an incidence of $127.8 \mathrm{BC}$ cases per 100,000 women and a mortality rate of 25.5 per 100,000 between 2000 and 2004 [5]. In addition, most patients are diagnosed under 50 years [6] and one fourth under 40 [7] and $15 \%$ present with distant or metastatic disease [4].

Many prognostic and predictive biomarkers are utilized in $\mathrm{BC}$, among which the estrogen receptor (ER), progesterone receptor (PR) and human epidermal growth factor receptor 2 (HER2) have been validated and are currently used in routine clinical practice for decision-making regarding specific treatments in $\mathrm{BC}[8]$.

HER2 gene is a proto-oncogene encoding the HER2 receptor. HER2 gene amplification has been identified in 25 to $30 \%$ of primary BCs [9]. Patients with HER2-positive disease are more likely to develop disease recurrence and to have a shorter overall survival (OS) and an overall poor prognosis, but these patients can benefit from specific anti-HER2 targeted therapies that can improve their outcomes [8] such as trastuzumab [10], lapatinib, pertuzumab, and other novel anti-HER2 targeted therapies [11].

As per the American Society of Clinical Oncology/ College of American Pathologists (ASCO/CAP) guidelines, HER2 testing is recommended in primary invasive and metastatic BCs. Tissue can be obtained through either core or excisional biopsies. Two tests are currently validated and used for HER2 status testing: immunohistochemistry (IHC) assay for HER2 protein overexpression and in situ hybridization (ISH) assay for HER2 gene amplification [12].

HER2 overexpression has been evaluated in several studies in Saudi Arabia. One study compared consecutive BC series from Switzerland and Saudi Arabia between 1988 and 2002 and reported a 31\% frequency of HER2 amplification in Saudi Arabian BCs, which was significantly higher than that reported in the Swiss samples (17\%) [13]. Another study reported a $28.3 \%$ rate of HER2 overexpression between 2000 and 2004 and found no correlation between HER2 status and other prognostics factors [14].

The results reported in the aforementioned studies were not always consistent. Moreover, data collected covered the period between 1988 until 2008 and most studies sampled only one center in Saudi Arabia. The number of drugs licensed for HER2 over-expressed BC is expanding across all treatment settings (neoadjuvant, adjuvant and palliative). Studying the frequency and the outcome of HER2 over-expressed BC can help health economists, funding agencies and decision makers draw better and more evidence-based strategies $[15,16]$.

The current study is structured in 2 parts utilizing combined data from three major centers in Saudi Arabia, to provide a greater representation of the frequency of HER2 overexpression in the Saudi Arabian BC population in part 1 . In addition, data collection covered the period extending from 2007 until 2013, to provide an updated and accurate estimation of the frequency of HER2 overexpression figures. In part 2 of the study, we aim to present an in-depth description of the clinic-pathological features, management and the clinical outcome of HER2-positive BC in Saudi Arabia.

\section{Methods}

This was a retrospective multicenter two-part study, consisting of chart review of newly diagnosed $\mathrm{BC}$ patients previously treated in routine clinical practice setting. The study was conducted in three pre-selected sites in Saudi Arabia: King Faisal Specialist Hospital and Research Center Gen.Org. (Jeddah), King Abdullah International Medical Research Center [KAIMRC]-King Abdulaziz Medical City (Riyadh), and King Fahad Specialist Hospital (Dammam). Data was collected between October 2016 and October 2018.

\section{Study part 1}

The first part of the study aimed to determine the frequency of HER2 overexpression and included male and female adult patients ( $>18$ years) residing in Saudi Arabia, who were newly diagnosed with early or advanced BC between 2007 and 2013, and who had available HER2 testing results. The only reason for exclusion was having an inconclusive or equivocal result of HER2 overexpression.

The pathology records of all eligible patients at the 3 pre-selected sites during the pre-defined period were retrospectively reviewed for HER2 status determination. HER2 overexpression was determined based on the treating physician's assessment of the pathology report regardless of the type of tissue sampled; HER2 testing methods included IHC and fluorescent in situ hybridization (FISH). 


\section{Study part 2}

In the second part of the study, a representative sample was selected from all patients with HER2-positive $\mathrm{BC}$ identified in part 1 of the study, and medical charts of this sample were retrospectively reviewed for the collection of data. The second part aimed to describe the demographic and clinical characteristics, as well as the hormone receptor status, treatment regimens, survival, response to treatment, and adverse events (AEs) in the sample of patients with HER2-positive disease only.

Data collected in the second part of the study included patient demographic and anthropometric characteristics (sex, age, nationality, body mass index [BMI]), site of tumor tissue sampling and HER2 testing method, ER and PR tests' results, patient clinico-pathologic profile (date of diagnosis, primary cancer site, BC stage and metastatic sites, performance status), and comorbidities. The treatment settings (neoadjuvant, adjuvant, and palliative setting) were also recorded. Additionally, data on radical radiotherapy, pharmacological therapy (regimens) and surgery was collected. Response to treatment and patient outcome expressed as progression-free survival [PFS] and overall survival [OS] was calculated. We also recorded the patients' status at cut-off point and safety data (only AEs reported in patients while receiving trastuzumab were collected).

\section{Study endpoints}

The primary endpoint was the frequency of HER2 overexpression in patients newly diagnosed with early or advanced BC in the 3 study sites.

Secondary endpoints were studied in a sample of HER2-positive BC patients and included:

- Patients' demographic and clinical characteristics

- The percentage of patients with ER/PR positive BC

- The type of treatment regimens used in the management of BC

- Median OS and PFS and the percentage of patients with complete response, partial response, progressive disease and stable disease

- Safety data (AEs reported in patients while receiving trastuzumab with or without other concomitant systemic therapies).

\section{Statistical methods \\ Sample size justification}

No pre-estimated sample size was provided for part 1 of the study. The determination of the frequency of HER2 overexpression was based on the review of pathology reports of all patients newly diagnosed with BC between 2007 and 2013 and who had available HER2 testing results in the pre-selected sites. For part 2 of the study, a representative sample from all HER2-positive BC patients was selected. The selection of these cases was based on the integrity and completeness of data. Patients who had significant missing information precluding meaningful analysis were excluded. Examples of such patients include those who transferred their care to another institution shortly after diagnosis, refused standard systemic anti-cancer therapy recommendations and those with missing or unobtainable medical records for any reason.

\section{Statistical analyses}

All statistical analyses were performed using IBM SPSS Statistics for Windows, Version 22.0. Armonk, NY: IBM Corp.

Qualitative variables were summarized using descriptive statistics and were reported as frequencies and percentages. Quantitative variables were presented as means and standard deviations. Confidence intervals were two sided and provided at the 95\% confidence level. Significance level was set at $5 \%$ and $p$-value $<0.05$ was considered as significant. No missing data were imputed. For the OS and PFS analyses, patients were classified based on the first treatment they received after diagnosis: curative intent treatment (neoadjuvant or adjuvant therapy) or palliative intent treatment (treatment for metastatic disease). Survival data were estimated by Kaplan Meier approach and graphically presented. Log Rank test (Mantel-Cox) was used to compare the survival distributions in patients on curative versus palliative treatment. Patients who had not received pharmacotherapy were excluded from the OS and PFS analyses, since they could not be categorized as palliative/curative. The frequency of AEs was summarized according to the System Organ Classification (SOC) and within each SOC, by MedDRA preferred term.

\section{Results \\ Study part 1}

From the 3 study sites, 1867 BC records with available HER2 testing results were included. HER2 was overexpressed in 559 patients (29.9\%) (Table 1). Of the 559 HER2-positive BC cases identified in part 1, 348 patients were included in part 2 of the study (Fig. 1).

\section{Study part 2}

The median age of patients was 46 years at diagnosis and 52 years at study inclusion. Only 3 patients were male $(0.9 \%)$, and the majority of patients were Saudi Arabian (92.5\%). Of the total of 348 patients, 139 (39.9\%) had a comorbidities, the most common of which were hypertension (22.7\%), diabetes mellitus 
Table 1 HER2 status in patients with available HER2 testing results between 2007 and 2013

\begin{tabular}{llll}
\hline $\mathbf{N}$ & Positive & Negative & Total \\
& $\mathbf{5 5 9}$ & $\mathbf{1 3 0 8}$ & $\mathbf{1 8 6 7}$ \\
\hline HER2 status with 95\% Cl & $29.9 \%(27.9-32.0 \%)$ & $70.1 \%(68.0-72.1 \%)$ & $100 \%$ \\
$\begin{array}{l}\text { Per site distribution: } n \text { (\%) } \\
\text { King Faisal Specialist Hospital and Research Center Gen. Org. (Jeddah) }\end{array}$ & $210(29.1 \%)$ & $511(70.9 \%)$ & $721(100 \%)$ \\
King Abdullah International Medical Research Center [KAIMRC] - King & $237(31.1 \%)$ & $526(68.9 \%)$ & $763(100 \%)$ \\
$\quad$ Abdulaziz Medical City (Riyadh) & $112(29.2 \%)$ & $271(70.8 \%)$ & $383(100 \%)$ \\
King Fahad Specialist Hospital (Dammam) & & & \\
\hline
\end{tabular}

Cl confidence interval

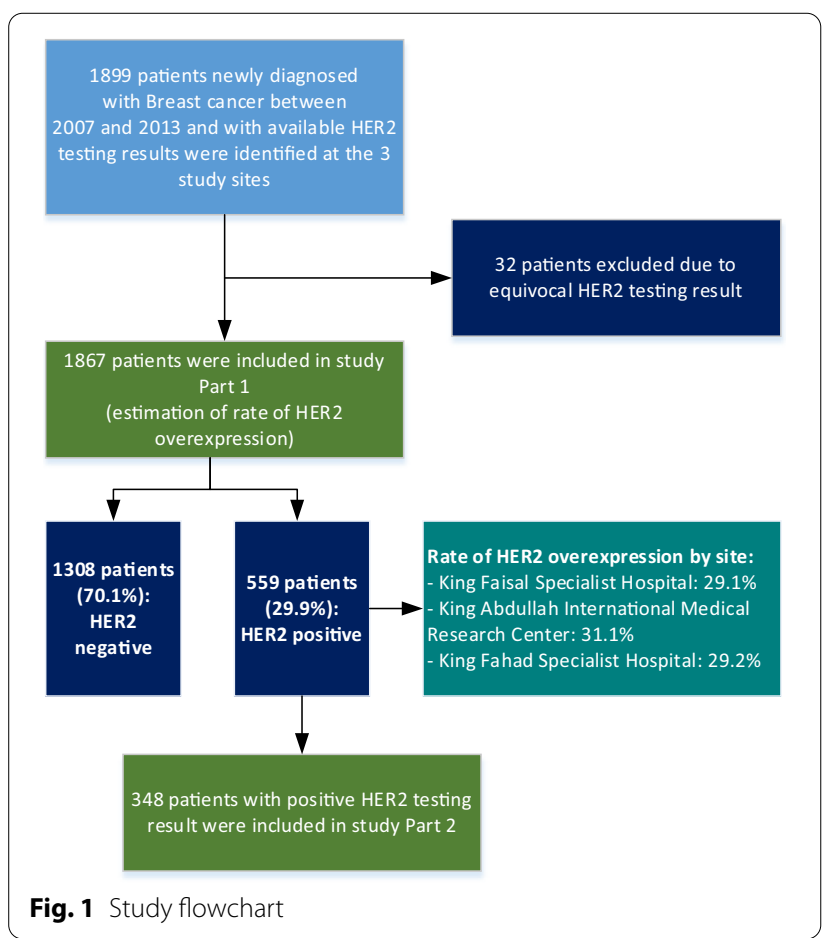

(22.1\%), dyslipidemia (18.7\%), hypothyroidism (9.5\%), and ischemic heart disease (4.1\%). In HER2-positive BC patients, $42.4 \%$ were ER and PR-negative (Table 2).

Based on the TNM classification at diagnosis, 21.9\% of patients had Stage III A, $18.1 \%$ had Stage III B, and $13.1 \%$ had Stage IV tumors. Data on site of biopsy and HER2 testing method, as well as primary cancer site, metastatic sites, and performance status are summarized in Table 2.

The majority of patients underwent curative intent surgery $(n=293 ; 84.2 \%)$ and $249(71.8 \%)$ received postoperative adjuvant radiotherapy. Average tumor size was $3.5 \pm 2.5 \mathrm{~cm}$. Infiltrating ductal carcinoma was the most common form of BC (92.9\%). Tumor margins were negative (clean, clear, not involved) in $92.5 \%$ of the cases. Details on tumor characteristics, lymph node status, and clinical response at last patient evaluation are present in Table 2.

As for pharmacological therapy, 110 patients received neoadjuvant pharmacotherapy, 153 received adjuvant pharmacotherapy, and 46 patients received palliative pharmacotherapy as their first treatment, while 39 patients did not receive pharmacotherapy during follow-up. Further details on pharmacotherapy in different treatment settings are present in Table 3.

The most commonly used regimen in neoadjuvant setting was Chemotherapy+ Trastuzumab combination (79.1\%), while in adjuvant setting, the most commonly used regimen was Hormonotherapy alone (56.2\%). As for palliative therapy, the most commonly used regimen was Chemotherapy + Targeted therapy combination (64.8\%).

At the last patient evaluation available at end of data collection, $36.9 \%$ of patients had complete response, while $33.2 \%$ had progressive disease.

305 patients were included in the OS analysis. Of those who started on curative intent pharmacotherapy (Neoadjuvant/Adjuvant setting; $\mathrm{n}=259$ ) after diagnosis, 16 patients died during follow-up. Median OS was not reached in this group. As for patients who started on palliative intent pharmacotherapy (treatment of metastatic disease; $\mathrm{n}=46$ ) after diagnosis, 16 patients died during follow-up and median OS was 64.7 months (Fig. 2).

302 patients were included in the PFS analysis. For those who started on curative intent pharmacotherapy (Neoadjuvant/Adjuvant setting; $\mathrm{n}=256$ ) after diagnosis, 57 patients had documented progression during followup. Median PFS was not reached in this group. As for patients who started on palliative intent pharmacotherapy (treatment of metastatic disease; $\mathrm{n}=46$ ) after diagnosis, 23 patients had documented progression during follow-up and median PFS was 29.3 months. The difference in PFS distribution between the 2 groups was highly significant $(\mathrm{p}<0.001)$ (Fig. 3).

17 patients were reported to have experienced SAEs (4.9\%) and 83 patients to have experienced AEs during the administration of trastuzumab. The most commonly 
Table 2 Demographic and clinical characteristics of HER2 positive Breast Cancer Patients

\begin{tabular}{ll}
\hline Demographic and clinical characteristics & Total \\
$\mathrm{N}=348$
\end{tabular}

Age at study inclusion (years), $n=348$

Mean \pm SD

Age at breast cancer diagnosis (years), $n=344$

Mean \pm SD

$\operatorname{Sex}, n=348$

Male

Female

Nationality, $n=348$

Saudi Arabian

Other

Body Mass Index $\left(\mathrm{kg} / \mathrm{m}^{2}\right), n=262$

Mean \pm SD

Breast cancer stage based on TNM classification at diagnosis, $n=260$

Stage I

Stage II

Stage III A

Stage III B

Stage IV

Metastatic sites, $n=30$

Bone

Lung

Liver

Lymph node

Brain

Ovaries

Peritoneum

Primary cancer site, $n=348$

Right

Left

Bilateral

Unknown

Performance status, $n=187$

Performance status $=0$

Performance status $=1$

Performance status $=2$

Performance status $=3$

Performance status $=4$

Performance status $=5$

Site of biopsy, $n=348$

Primary tumor site

Metastatic tumor site

Unknown, not recorded

HER2 test method, $n=348$

$\mathrm{FISH}$

IHC

Curative intent surgery performed, $n=348$

Yes

No

$48.14 \pm 11.67$

$3(0.9 \%)$

$345(99.1 \%)$

$322(92.5 \%)$

$26(7.5 \%)$

$103(39.6 \%)$

$57(21.9 \%)$

$47(18.1 \%)$

$34(13.1 \%)$

$15(50.0 \%)$

$13(43.3 \%)$

$13(43.3 \%)$

$4(13.3 \%)$

$3(10.0 \%)$

$1(3.3 \%)$

$1(3.3 \%)$

$175(50.3 \%)$

$164(47.1 \%)$

$8(2.3 \%)$

$1(0.3 \%)$

$20(10.7 \%)$

$122(65.2 \%)$

$31(16.6 \%)$

$9(4.8 \%)$

$5(2.7 \%)$

$0(0.0 \%)$

$328(94.3 \%)$

$15(4.3 \%)$

$5(1.4 \%)$

$53(15.3 \%)$

$294(84.7 \%)$

$293(84.2 \%)$

$55(15.8 \%)$
Table 2 (continued)

Demographic and clinical characteristics

Total

$\mathrm{N}=348$

If Yes, tumor size $(\mathrm{cm}), n=264$

$\begin{array}{ll}\text { Mean } \pm \text { SD } & 3.5 \pm 2.5\end{array}$

$54.07 \pm 11.55 \quad$ Breast cancer pathology, $n=283$

Infiltrating ductal carcinoma

$263(92.9 \%)$

Infiltrating lobular carcinoma

$0(0.0 \%)$

Medullary carcinoma

$0(0.0 \%)$

Inflammatory breast cancer

$2(0.7 \%)$

Tubular carcinoma

$1(0.4 \%)$

Mucinous carcinoma

$2(0.7 \%)$

Ductal carcinoma in situ

$13(4.6 \%)$

Invasive ductal carcinoma

$2(0.7 \%)$

Hormone-receptor status, $n=340$

ER-/PR-

$144(42.4 \%)$

$E R+$ and/or PR +

$196(57.6 \%)$

Tumor margins, $n=265$

Negative

$245(92.5 \%)$

Positive

$20(7.5 \%)$

More than one tumor in the breast, $n=346$

Yes

$58(16.8 \%)$

No

$288(83.2 \%)$

If Yes, tumor origin, $n=53$

Multifocal breast cancer

$33(62.3 \%)$

Multicentric breast cancer

$20(37.7 \%)$

Lymphovascular invasion, $n=289$

Yes

$121(41.9 \%)$

No

$168(58.3 \%)$

Axillary lymph nodes examined (sentinel, sampling, dissection), $n=313$

Yes

$256(81.8 \%)$

No

$57(18.2 \%)$

Number of lymph nodes examined, $n=254$

Mean \pm SD

$14.5 \pm 11.2$

Number of lymph nodes involved, $n=253$

Mean \pm SD

$3.7 \pm 7.6$

Clinical response at last patient evaluation, $n=217$

Stable disease

$61(28.1 \%)$

Progressive disease

$72(33.2 \%)$

Partial response

$4(1.8 \%)$

Complete response

$80(36.9 \%)$

$S D$ standard deviation, FISH fluorescent in situ hybridization, $I H C$

ImmunoHistoChemistry, ER estrogen receptor, $P R$ progesterone receptor

reported AEs were decreased ejection fraction (7.2\%), stomatitis $(3.7 \%)$, and vomiting (2.9\%). Other AEs are detailed in Table 4.

\section{Discussion}

Data on HER2 overexpression in Saudi Arabia reported in the literature mainly covers the period from 1988 until 2008. This study provided updated figures on the rate of 
Table 3 Pharmacological treatments and radiotherapy in HER2 positive Breast Cancer Patients

\begin{tabular}{|c|c|}
\hline & n (\%) \\
\hline \multicolumn{2}{|c|}{$\begin{array}{l}\text { Pharmacological treatment setting from diagnosis till end of data collec- } \\
\text { tion (cut-off point), } n=309\end{array}$} \\
\hline Neoadjuvant only & $10(3.2 \%)$ \\
\hline Adjuvant only & $134(43.4 \%)$ \\
\hline Metastatic only & $46(14.9 \%)$ \\
\hline Neoadjuvant + Adjuvant + Metastatic & $22(7.1 \%)$ \\
\hline Neoadjuvant + Adjuvant & $74(23.9 \%)$ \\
\hline Adjuvant + Metastatic & $19(6.1 \%)$ \\
\hline \multicolumn{2}{|l|}{ Neoadjuvant treatment regimens, $n=110$} \\
\hline Neoadjuvant + Metastatic & $4(1.3 \%)$ \\
\hline Hormonotherapy alone & $3(2.7 \%)$ \\
\hline Chemotherapy alone & $83(75.5 \%)$ \\
\hline Trastuzumab alone & $7(6.4 \%)$ \\
\hline Chemotherapy + Trastuzumab & $87(79.1 \%)$ \\
\hline Other regimens & $2(1.8 \%)$ \\
\hline \multicolumn{2}{|l|}{ Adjuvant treatment regimens, $n=249$} \\
\hline Hormonotherapy alone & $140(56.2 \%)$ \\
\hline Chemotherapy alone & $113(45.4 \%)$ \\
\hline Trastuzumab alone & $96(38.6 \%)$ \\
\hline Chemotherapy + Trastuzumab & $129(51.8 \%)$ \\
\hline Hormonotherapy + Trastuzumab & $22(8.8 \%)$ \\
\hline \multicolumn{2}{|l|}{ Metastatic treatment regimens, $n=91$} \\
\hline Hormonotherapy alone & $41(45.1 \%)$ \\
\hline Chemotherapy alone & $40(44.0 \%)$ \\
\hline Targeted therapy alone & $36(45.1 \%)$ \\
\hline Chemotherapy + Targeted therapy & $59(64.8 \%)$ \\
\hline Hormonotherapy + Targeted therapy & $14(15.4 \%)$ \\
\hline Hormonotherapy + Chemotherapy + Targeted therapy & $2(3.3 \%)$ \\
\hline \multicolumn{2}{|l|}{ Radiotherapy, $n=347$} \\
\hline No & $98(28.2 \%)$ \\
\hline Yes & $249(71.8 \%)$ \\
\hline
\end{tabular}

HER2 overexpression in the Saudi Arabian BC population, for the period between 2007 and 2013.

HER2 overexpression rate was found to be $29.9 \%$ in our sample of newly diagnosed BC patients. The American Cancer Society has reported the HER2 overexpression rate in the USA to be $17 \%$ in 2017-2018 [17], while several studies in Middle Eastern and Arab populations suggest much higher rates in those populations. AlTamimi et al. reported a HER2 overexpression rate of $28 \%$ in a sample of Saudi Arabian women [18]. Alnegheimish et al. reported a HER2 overexpression rate of $26.8 \%$ in a retrospective study done in Riyadh between 2010 and 2014 [19]. Other studies such as Chahine et al. reported even higher rates (39.7\%) in Lebanese patients between 2008 and 2013 [20]. One possible explanation for the higher HER2 overexpression rate in our study, beside potential ethnic variability, is the younger age at presentation for BC patients in our region. HER2 overexpression has been suggested to be associated with younger patient age in several studies [21-23].

As for age at diagnosis, the mean age of 48 years reported in our study is in line with numerous studies which reported similar values, among which is Saggu et al. [24] who reported a mean age of 49 years, using data from the Saudi Cancer Registry (1990-2010), in addition to other studies conducted in 2012-2014 and who have reported the same mean age of 48-50 years at diagnosis [7,25]. Averages reported in the US and Europe are more than 10 years above this number [26]. In the United States, $65.1 \%$ of BC cases occur in women older than 55 years, based on SEER data for 2001-2005 [27]. Najjar et al. attributed this difference to social, economic and population differences between Arab and Western populations [28]. This younger age at diagnosis should encourage Arab countries, including Saudi Arabia, to reconsider the age range of the target population of national BC screening programs.

Regarding hormone-receptor status, our study has reported that $54 \%$ of HER2-positive patients were also ER-positive, while $42 \%$ were ER and PR-negative. Rudat et al. has reported higher rates of ER-positive tumors (67\%), however, that study has examined hormonereceptor status regardless of HER2 receptor status [7]. Mohamed et al. [29] reported that $38 \%$ of patients with HER2-positive tumors were hormone-receptor negative, which is similar to the rate reported in our study.

Stage of disease at diagnosis was reported to be $39 \%$ and $13 \%$ for stages 3 and 4 in this study. Rudat et al. reported that $40 \%$ and $9 \%$ of BC patients at a tertiary care center in the Eastern province of Saudi Arabia had stage 3 and 4 tumors respectively [7], while another study reported that $30 \%$ and $16 \%$ of BC tumors in Saudi Arabia were stages 3 and 4 tumors respectively [26].

In our sample of HER2-positive BC patients, $84 \%$ and $72 \%$ of patients underwent curative intent surgery and postoperative adjuvant radiotherapy respectively. However, the type of surgery was not specified (breast-conserving or radical mastectomy) in our study, which might preclude comparison to other figures reported in similar studies.

The large tumor size at diagnosis observed in this study $(3.5 \mathrm{~cm})$ implies that tumors are being diagnosed later than should be, which might be related to insufficient public awareness on the importance of periodic clinical and self-examination of the breasts. More efforts should be made to improve the efficacy and reachability of national screening programs, to ensure early diagnosis and most favorable patient outcomes. 


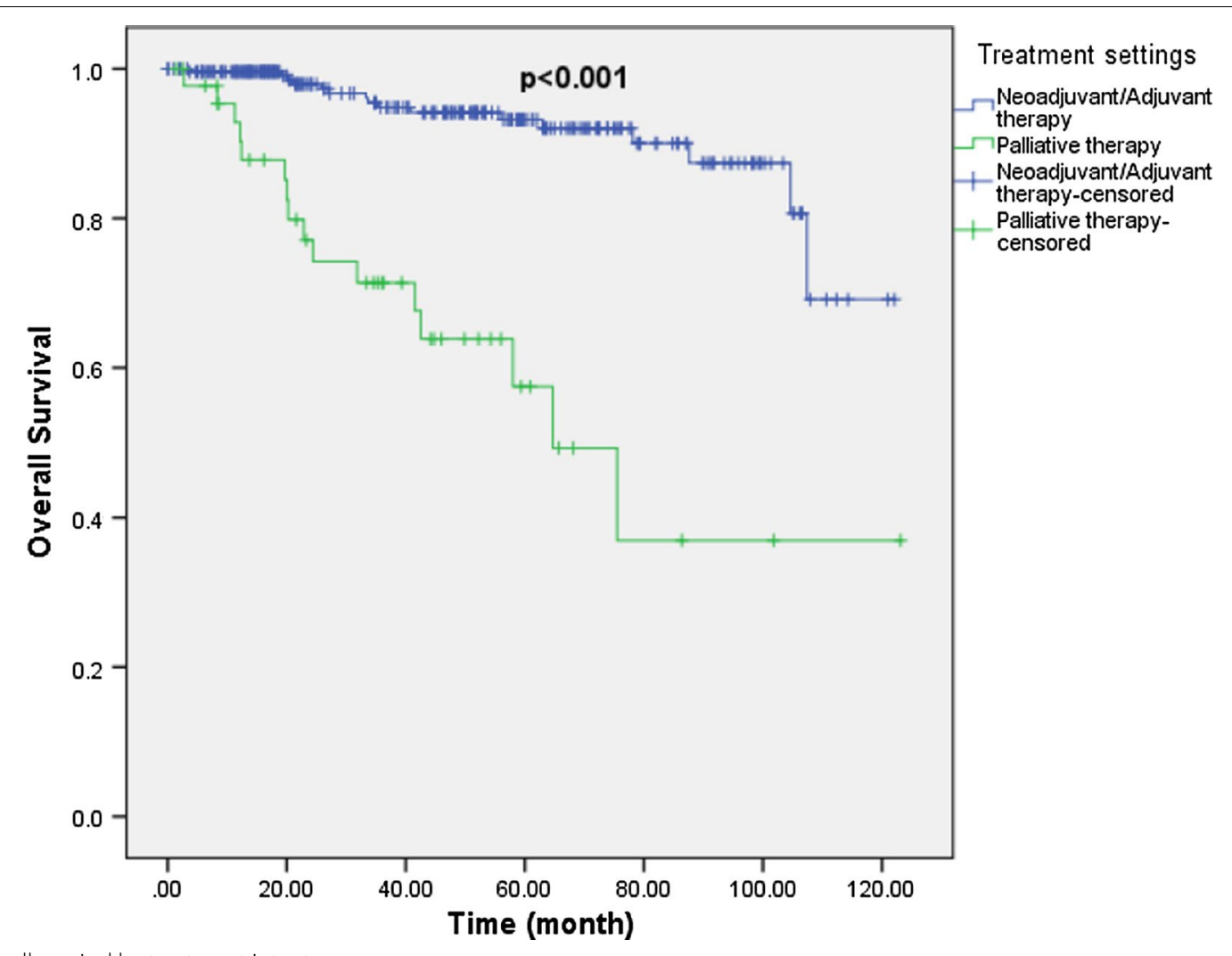

Fig. 2 Overall survival by treatment intent

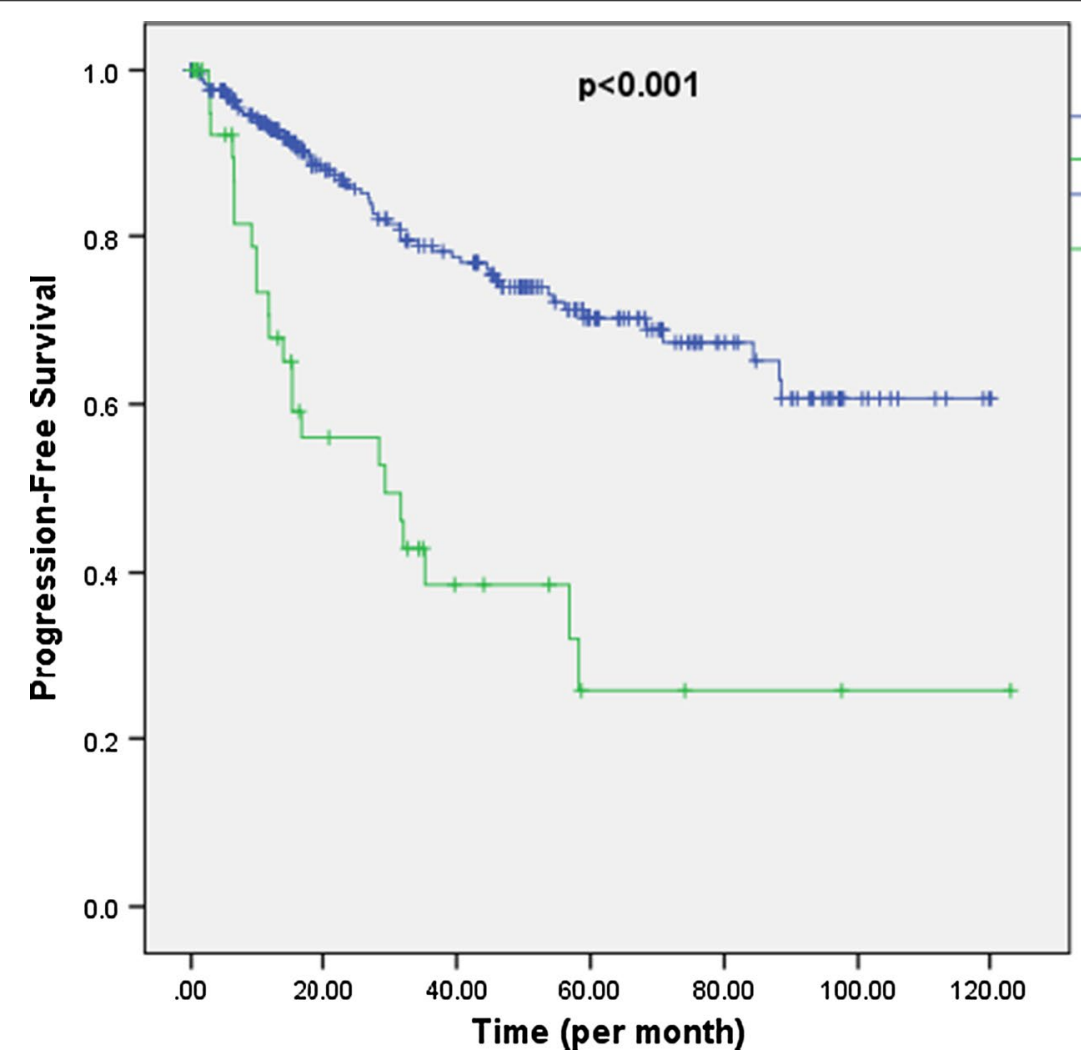

Treatment settings

Neoadjuvant/Adjuvant

therapy

Palliative therapy

Neoadjuvant/Adjuvant

therapy-censored

Palliative therapy-

censored

Fig. 3 Progression-free survival by treatment intent 
Table 4 Adverse events experienced during trastuzumab treatment in patients with HER2-positive breast cancer

System organ class
MedDRA preferred term

\begin{tabular}{|c|c|}
\hline \multicolumn{2}{|l|}{ Any system organ class } \\
\hline Total & $83(23.9 \%)$ \\
\hline Investigations & $24(6.9 \%)$ \\
\hline Ejection fraction decreased & $25(7.2 \%)$ \\
\hline Hepatic enzymes increased & $1(0.3 \%)$ \\
\hline Blood and lymphatic system disorders & $15(4.3 \%)$ \\
\hline Neutropenia & $9(2.6 \%)$ \\
\hline Febrile neutropenia & $8(2.3 \%)$ \\
\hline Lymphedema & $1(0.3 \%)$ \\
\hline Gastrointestinal disorders & $15(4.3 \%)$ \\
\hline Stomatitis & $13(3.7 \%)$ \\
\hline Vomiting & $10(2.9 \%)$ \\
\hline Abdominal pain upper & $2(0.6 \%)$ \\
\hline Abdominal pain & $1(0.3 \%)$ \\
\hline Dysphagia & $1(0.3 \%)$ \\
\hline Gastritis & $1(0.3 \%)$ \\
\hline Loose tooth & $1(0.3 \%)$ \\
\hline General disorders and administration site conditions & $14(4.0 \%)$ \\
\hline Pyrexia & $9(2.6 \%)$ \\
\hline Pain & $5(1.4 \%)$ \\
\hline Fatigue & $1(0.3 \%)$ \\
\hline Cardiac disorders & $16(4.6 \%)$ \\
\hline Cardiomyopathy & $8(2.3 \%)$ \\
\hline Dyspnea & $6(1.7 \%)$ \\
\hline Cardiac failure congestive & $2(0.6 \%)$ \\
\hline Bradycardia & $1(0.3 \%)$ \\
\hline Congestive cardiomyopathy & $1(0.3 \%)$ \\
\hline Palpitations & $1(0.3 \%)$ \\
\hline Nervous system disorders & $9(2.6 \%)$ \\
\hline Neuropathy peripheral & $6(1.7 \%)$ \\
\hline Dizziness & $2(0.6 \%)$ \\
\hline Hypoesthesia & $1(0.3 \%)$ \\
\hline Muscular weakness & $1(0.3 \%)$ \\
\hline Musculoskeletal and connective tissue disorders & $7(2.0 \%)$ \\
\hline Pain in extremity & $5(1.4 \%)$ \\
\hline Arthralgia & $2(0.6 \%)$ \\
\hline Back pain & $1(0.3 \%)$ \\
\hline Bone pain & $1(0.3 \%)$ \\
\hline Respiratory, thoracic and mediastinal disorders & $6(1.7 \%)$ \\
\hline Cough & $3(0.9 \%)$ \\
\hline Pleural effusion & $1(0.3 \%)$ \\
\hline Pneumonitis & $1(0.3 \%)$ \\
\hline Pulmonary embolism & $1(0.3 \%)$ \\
\hline Infections and infestations & $4(1.1 \%)$ \\
\hline Abscess limb & $1(0.3 \%)$ \\
\hline Bronchitis & $1(0.3 \%)$ \\
\hline Cellulitis & $1(0.3 \%)$ \\
\hline Respiratory tract infection & $1(0.3 \%)$ \\
\hline
\end{tabular}

Table 4 (continued)

\begin{tabular}{ll}
\hline System organ class & $\mathbf{n}(\%)$ \\
MedDRA preferred term & \\
\hline Injury, poisoning and procedural complications & $4(1.1 \%)$ \\
Cardiotoxicity & $4(1.1 \%)$ \\
Skin and subcutaneous tissue disorders & $4(1.1 \%)$ \\
Alopecia & $1(0.3 \%)$ \\
Dry skin & $1(0.3 \%)$ \\
Pigmentation disorder & $1(0.3 \%)$ \\
Pruritus & $1(0.3 \%)$ \\
Skin lesion & $1(0.3 \%)$ \\
Immune system disorders & $3(0.9 \%)$ \\
Hypersensitivity & $2(0.6 \%)$ \\
Skin reaction & $1(0.3 \%)$ \\
Renal and urinary disorders & $3(0.9 \%)$ \\
Flank pain & $2(0.6 \%)$ \\
Dysuria & $1(0.3 \%)$ \\
Urinary tract infection & $1(0.3 \%)$ \\
Reproductive system and breast disorders & $2(0.6 \%)$ \\
Endometrial hyperplasia & $1(0.3 \%)$ \\
Hematuria & $1(0.3 \%)$ \\
Ear and labyrinth disorders & $1(0.3 \%)$ \\
Otitis externa & $1(0.3 \%)$ \\
Metabolism and nutrition disorders & $1(0.3 \%)$ \\
Decreased appetite & $1(0.3 \%)$ \\
Vascular disorders & $1(0.3 \%)$ \\
Hot flush & $1(0.3 \%)$ \\
\hline Frequency of AEs is sorted in descending order in the total column & \\
A patient with multiple occurrences of an AE for a preferred term or system & \\
organ class is counted only once & \\
A patient with multiple AEs within a primary system organ class is counted only \\
once in the total row
\end{tabular}

Infiltrating ductal carcinoma is the most common type of $\mathrm{BC}$, despite the difference in exact rates reported in different studies. Our study has found that $93 \%$ of HER2positive patients had infiltrating ductal carcinoma, a rate that is higher than that reported in the Saudi Arabian 2015 Cancer Incidence Report (79\%) [4]. Albasri et al. [25] has reported an $85 \%$ rate of infiltrating ductal carcinoma in a study conducted in Al-Madinah region, while Rudat et al. [7] has reported a rate of $91 \%$ in a similar study.

Considering that this was a retrospective study relying on secondary data collection, the data obtained may be subject to selection bias, since many patients could have been excluded due to the unavailability of HER2 testing results. In addition, the retrospective design might have affected the completeness of the data collected, due to the potential for missing data. Efforts to minimize bias and maximize data collection were done through selecting centers known to have high quality of clinical 
records-keeping. The comparison between HER2-positive and negative $\mathrm{BC}$ patients' characteristics was not possible due to the lack of a control arm in this study.

This study is of primary importance since it included patients from 3 different centers distributed all over Saudi Arabia, which allows for a more representative assessment of the actual frequency of HER2 overexpression in the general $\mathrm{BC}$ patients pool, considering that most previous studies on $\mathrm{BC}$ epidemiology were done in limited regions in Saudi Arabia. The large sample size of this study and the long follow-up duration further enhanced the study's representativeness. Our study explored the size of the burden of HER2 over-expressed BC diagnosed by traditional IHC and FISH and identified as a specific subgroup of patients that need to be managed accordingly. More genetically identified subgroups are likely to be identified in the future when modern techniques such as whole exome and RNA sequencing become established in routine clinical practice [30].

\section{Conclusions}

This study has provided updated figures regarding HER2 overexpression in BC patients in Saudi Arabia: HER2 overexpression rate (29.9\%) was within the range reported in previous studies conducted in Saudi Arabia. In addition, patients' demographic and clinical characteristics in this study were also similar to those reported earlier, with a median age at diagnosis of 46 years and one third of patients being diagnosed with locally advanced/ metastatic disease. This study has also highlighted prescription trends and survival outcomes in a sample of unique patients in Saudi Arabia.

\begin{abstract}
Abbreviations
BC: Breast cancer; ER: Estrogen receptor; PR: Progesterone receptor; HER2: Human epidermal growth factor receptor 2; ASCO/CAP: American Society of Clinical Oncology/College of American Pathologists; OS: Overall survival; IHC: ImmunoHistoChemistry; ISH: In situ hybridization; FISH: Fluorescent in situ hybridization; AEs: Adverse events; BMI: Body Mass Index; PFS: Progression-free survival; SOC: System organ classification; IRB: Institutional Review Board.
\end{abstract}

\begin{abstract}
Acknowledgements
In addition to the authors, we would like to thank the below sub-investigators and site coordinators for their contribution to the study: King Faisal Specialist Hospital and Research Center Gen.Org. (Jeddah). Ehab Mosaad (Sub-investigator), Eman Bawazeer (Site coordinator), Rawan Al Saedi (Site coordinator), Haneen Al Omar (Site coordinator). King Abdullah International Medical Research Center [KAIMRC], King Abdulaziz Medical City (Riyadh). Ahmed Hashim (Sub-investigator), Wael El-Melouk (Sub-investigator), Alaa Ali (Sub-investigator), Haytham Hamdan (Sub-investigator), Mohammed Alkaiyat (Site coordinator), Ahmad Al-Qudimat (Site coordinator). King Fahad Specialist Hospital (Dammam). Ahmad Shaheen (Sub-investigator), Zakaria Basbous (Site coordinator), Lamees Algain (Site coordinator), Amina Bukhamsin (Site coordinator), Reem Gaw (Site coordinator).
\end{abstract}

\section{Authors' contributions}

$J Z, A S$, and $\mathrm{HA}$ contributed to the study concept. All authors read and approved the final manuscript.

\section{Funding}

This study was sponsored by Roche Products Saudi Arabia. It was responsible for study design. However, it had no role in data collection, analysis, and interpretation. Medical writing assistance was funded by Roche Products Saudi Arabia.

\section{Availability of data and materials}

The data that support the findings of this study are available from [Clinserv CRO] but restrictions apply to the availability of these data, which were used under license for the current study, and so are not publicly available. Data are however available from the authors upon reasonable request and with permission of [Clinserv CRO].

\section{Ethics approval and consent to participate}

The study protocol was approved by the Institutional Review Boards (IRBs) of the 3 study sites: King Faisal Specialist Hospital and Research Center Gen. Org. (Jeddah) [Reference number: RC-J/301/37], King Abdullah International Medical Research Center [KAIMRC] —King Abdulaziz Medical City (Riyadh) [Reference number: IRBC/881/16], and King Fahad Specialist Hospital (Dammam) [Reference number: IRB-ONC0336]. In case of a request for accessing the medical records at any of the study sites, a written approval from the IRB of that site would be needed as per each IRB's requirements.

\section{Consent for publication}

Not applicable.

\section{Competing interests}

The authors declare that they have no competing interests.

\section{Author details}

${ }^{1}$ King Faisal Specialist Hospital and Research Centre, College of Medicine, Alfaisal University, Jeddah, Saudi Arabia. ${ }^{2}$ King Abdulaziz Medical City, Ministry of National Guard, Health Affairs, Riyadh, Saudi Arabia. ${ }^{3}$ King Fahad Specialist Hospital, Dammam, Saudi Arabia.

Received: 20 July 2020 Accepted: 23 December 2020

Published online: 06 January 2021

\section{References}

1. Bray F, Ferlay J, Soerjomataram I, Siegel RL, Torre LA, Jemal A. Global cancer statistics 2018: GLOBOCAN estimates of incidence and mortality worldwide for 36 cancers in 185 countries. CA Cancer J Clin. 2018;68:394424. https://doi.org/10.3322/caac.21492.

2. Rodríguez-Cuevas S, Macías CG, Franceschi D, Labastida S. Breast carcinoma presents a decade earlier in Mexican women than in women in the United States or European countries. Cancer. 2001;91:863-8.

3. El Saghir NS, Khalil MK, Eid T, El Kinge AR, Charafeddine M, Geara F, et al. Trends in epidemiology and management of breast cancer in developing Arab countries: a literature and registry analysis. Int J Surg. 2007;5:225-33. https://doi.org/10.1016/j.ijsu.2006.06.015.

4. Saudi Health Council. Cancer Incidence Report Saudi Arabia. 2015. https ://nhic.gov.sa/eServices/Documents/E\%20SCR\%20final\%206\%20NOV .pdf. Accessed 20 July 2020.

5. Al Diab A, Qureshi S, Al Saleh KA, Al Qahtani AH, Aleem A, Algamdi $M A$, et al. Review on breast cancer in the Kingdom of Saudi Arabia. Middle-East J Sci Res. 2013;14:532-43. https://doi.org/10.5829/idosi.mejsr .2013.14.4.7327

6. Ezzat AA, Ibrahim EM, Raja MA, Al-Sobhi S, Rostom A, Stuart RK. Locally advanced breast cancer in Saudi Arabia: high frequency of stage III in a young population. Med Oncol. 1999;16:95-103. https://doi.org/10.1007/ BF02785842.

7. Rudat V, Brune-Erbe I, Noureldin A, Bushnag Z, Almuraikhi N, Altuwaijri S. Epidemiology of breast cancer patients at a tertiary care center in the Eastern Province of Saudi Arabia. Gulf J Oncolog. 2012;1:45-9.

8. Weigel MT, Dowsett M. Current and emerging biomarkers in breast cancer: prognosis and prediction. Endocr Relat Cancer. 2010;17:R245-62. https://doi.org/10.1677/ERC-10-0136.

9. Slamon DJ, Godolphin W, Jones LA, Holt JA, Wong SG, Keith DE, et al. Studies of the HER-2/neu proto-oncogene in human breast and ovarian 
cancer. Science. 1989;244:707-12. https://doi.org/10.1126/science.24701 52.

10. Dawood S, Broglio K, Buzdar AU, Hortobagyi GN, Giordano SH. Prognosis of women with metastatic breast cancer by HER2 status and trastuzumab treatment: an institutional-based review. J Clin Oncol. 2010;28:92-8. https ://doi.org/10.1200/JCO.2008.19.9844.

11. Ross JS, Slodkowska EA, Symmans WF, Pusztai L, Ravdin PM, Hortobagyi GN. The HER-2 receptor and breast cancer: ten years of targeted antiHER-2 therapy and personalized medicine. Oncologist. 2009;14:320-68. https://doi.org/10.1634/theoncologist.2008-0230.

12. Wolff AC, Hammond ME, Hicks DG, Dowsett M, McShane LM, Allison KH et al. Recommendations for human epidermal growth factor receptor 2 testing in breast cancer: American Society of Clinical Oncology/College of American Pathologists clinical practice guideline update. J Clin Oncol. 2013;31:3997-4013. https://doi.org/10.1200/JCO.2013.50.9984.

13. Al-Kuraya K, Schraml P, Sheikh S, Amr S, Torhorst J, Tapia C, et al. Predominance of high-grade pathway in breast cancer development of Middle East women. Mod Pathol. 2005;18:891-7. https://doi.org/10.1038/modpa thol.3800408.

14. Al-Ahwal MS. HER-2 positivity and correlations with other histopathologic features in breast cancer patients-hospital based study. J Pak Med Assoc. 2006:56:65-8.

15. Jacobs VR, Bogner G, Schausberger CE, Reitsamer R, Fischer T. Relevance of health economics in breast cancer treatment: integration of economics in the management of breast cancer at the clinic level. Breast Care (Basel). 2013;8(1):7-14. https://doi.org/10.1159/000348370.

16. Barrios $\mathrm{CH}$, Reinert T, Werutsky $\mathrm{G}$. Access to high-cost drugs for advanced breast cancer in Latin America, particularly trastuzumab. Ecancermedicalscience. 2019;13:898. https://doi.org/10.3332/ecancer.2019.898.

17. American Cancer Society. Breast Cancer Facts \& Figures 2017-2018. https ://www.cancer.org/content/dam/cancer-org/research/cancer-facts-andstatistics/breast-cancer-facts-and-figures/breast-cancer-facts-and-figur es-2017-2018.pdf. Accessed 20 July 2020.

18. Al-Tamimi DM, Bernard PS, Shawarby MA, Al-Amri AM, Hadi MA. Distribution of molecular breast cancer subtypes in middle eastern-Saudi Arabian women: a pilot study. Ultrastruct Pathol. 2009;33:141-50. https://doi. org/10.1080/01913120903183135.

19. Alnegheimish NA, Alshatwi RA, Alhefdhi RM, Arafah MM, AlRikabi AC, Husain S. Molecular subtypes of breast carcinoma in Saudi Arabia. A retrospective study. Saudi Med J. 2016;37:506-12. https://doi.org/10.15537/ smj.2016.5.15000.
20. Chahine G, El Rassy E, Khazzaka A, Saleh K, Rassy N, Khalife N, et al. Characteristics of incident female breast cancer in Lebanon, 1990-2013: descriptive study of 612 cases from a hospital tumor registry. Cancer Epidemiol. 2015;39:303-6. https://doi.org/10.1016/j.canep.2015.03.008.

21. Fourati A, Boussen H, El May MV, Goucha A, Dabbabi B, Gamoudi A, et al. Descriptive analysis of molecular subtypes in Tunisian breast cancer. Asia Pac J Clin Oncol. 2014;10:e69-74. https://doi.org/10.1111/ajco.12034.

22. AIZaman AS, Mughal SA, AIZaman YS, AIZaman ES. Correlation between hormone receptor status and age, and its prognostic implications in breast cancer patients in Bahrain. Saudi Med J. 2016;37:37-42. https://doi. org/10.15537/smj.2016.1.13016.

23. Shawarby MA, Al-Tamimi DM, Ahmed A. Molecular classification of breast cancer: an overview with emphasis on ethnic variations and future perspectives. Saudi J Med Med Sci. 2013;1:14-9. https://doi. org/10.4103/1658-631X.112908.

24. Saggu S, Rehman H, Abbas ZK, Ansari AA. Recent incidence and descriptive epidemiological survey of breast cancer in Saudi Arabia. Saudi Med J. 2015;36:1176-80. https://doi.org/10.15537/smj.2015.10.12268.

25. Albasri A, Hussainy AS, Sundkji I, Alhujaily A. Histopathological features of breast cancer in Al-Madinah region of Saudi Arabia. Saudi Med J. 2014;35:1489-93

26. Ibrahim EM, Al-Mulhim FA, Al-Amri A, Al-Muhanna FA, Ezzat AA, Stuart RK, et al. Breast cancer in the eastern province of Saudi Arabia. Med Oncol. 1998;15:241-7. https://doi.org/10.1007/BF02787207.

27. National Cancer Institute. SEER Cancer Statistics Review 1975-2005 http://seer.cancer.gov/archive/csr/1975_2005/. Accessed 20 July 2020.

28. Najjar H, Easson A. Age at diagnosis of breast cancer in Arab nations. Int J Surg. 2010;8:448-52. https://doi.org/10.1016/j.jijsu.2010.05.012.

29. Mohammed RAA, Radwan MEM, Alrufayi BM, Abdulaziz LAQ. Does loss of hormonal receptors influence the pathophysiological characteristics of the HER-2 breast cancer phenotype? Pathophysiology. 2018;25:439-44. https://doi.org/10.1016/j.pathophys.2018.09.004.

30. Kosvyra A, Maramis C, Chouvarda I. Developing an integrated genomic profile for cancer patients with the use of NGS data. Emerg Sci J. 2019;3(3):157-67. https://doi.org/10.28991/esj-2019-01178.

\section{Publisher's Note}

Springer Nature remains neutral with regard to jurisdictional claims in published maps and institutional affiliations.
Ready to submit your research? Choose BMC and benefit from:

- fast, convenient online submission

- thorough peer review by experienced researchers in your field

- rapid publication on acceptance

- support for research data, including large and complex data types

- gold Open Access which fosters wider collaboration and increased citations

- maximum visibility for your research: over $100 \mathrm{M}$ website views per year

At BMC, research is always in progress.

Learn more biomedcentral.com/submissions 